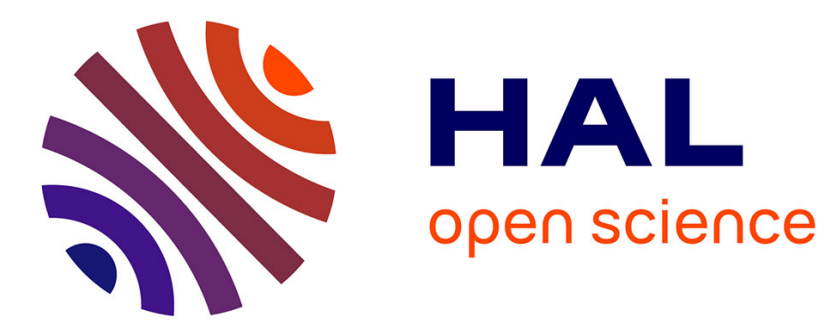

\title{
Short Bragg mirrors with adiabatic modal conversion
}

David Peyrade, Eric Silberstein, Philippe Lalanne, Anne Talneau, Yong Chen

\section{To cite this version:}

David Peyrade, Eric Silberstein, Philippe Lalanne, Anne Talneau, Yong Chen. Short Bragg mirrors with adiabatic modal conversion. Applied Physics Letters, 2002, 81 (2), pp.829-831. 10.1063/1.1497722 . hal-00878129

\section{HAL Id: hal-00878129 \\ https://hal-iogs.archives-ouvertes.fr/hal-00878129}

Submitted on 29 Oct 2013

HAL is a multi-disciplinary open access archive for the deposit and dissemination of scientific research documents, whether they are published or not. The documents may come from teaching and research institutions in France or abroad, or from public or private research centers.
L'archive ouverte pluridisciplinaire HAL, est destinée au dépôt et à la diffusion de documents scientifiques de niveau recherche, publiés ou non, émanant des établissements d'enseignement et de recherche français ou étrangers, des laboratoires publics ou privés. 


\title{
Short Bragg mirrors with adiabatic modal conversion
}

\author{
D. Peyrade \\ Laboratoire de Photonique et de Nanostructures, Centre National de la Recherche Scientifique, \\ Route de Nozay, 91460 Marcoussis, France
}

E. Silberstein and Ph. Lalanne ${ }^{\mathrm{a})}$

Laboratoire Charles Fabry de l'Institut Optique, Centre National de la Recherche Scientifique et Université Paris Sud, BP 147, 91403 Orsay Cedex, France

A. Talneau and Y. Chen

Laboratoire de Photonique et de Nanostructures, Centre National de la Recherche Scientifique, Route de Nozay, 91460 Marcoussis, France

(Received 22 April 2002; accepted for publication 12 June 2002)

\begin{abstract}
A silicon-on-insulator waveguide-based microcavity with short tapers has been realized by incorporating two identical eight-groove tapers at the two reflector extremities. The microstructure has an overall length of $14 \mu \mathrm{m}$ and consists of two first-order Bragg mirrors with narrow slits $(90$ $\mathrm{nm}$ ) and of four series of slits with progressively varying widths (tapers). A comparison with a nontapered cavity evidences the beneficial effect of the taper, a lowering of the radiation losses.

(C) 2002 American Institute of Physics. [DOI: 10.1063/1.1497722]
\end{abstract}

Many important devices use periodic microstructures of alternating layers of dielectric materials to enhance reflection. Usually, the refractive index contrast of dielectric layers is low, typically $1 \%$ in a distributed Bragg reflector, and a large number of small reflections over a long propagation distance are needed to warrant high reflectivity. ${ }^{1}$ Alternatively, periodic microstructures deeply etched into a semiconductor waveguide offer high refractive-index contrasts and much shorter interaction lengths. Examples include photonic wires or air-bridge microcavities ${ }^{2,3}$ and very compact Bragg reflectors. ${ }^{4-6}$ Responding to the quest for miniaturization in optoelectronics, these new mirrors implemented in short cavities additionally offer interesting perspectives: Large free spectral ranges, small modal volumes as is required for controlling the spontaneous emission of atoms in the cavity, and low threshold lasers.

For weak refractive-index modulations, nearly $100 \%$ modal reflectivity is achieved for long enough mirrors, thus providing a lossless operation for the mirror. For strong corrugations, the Bragg mirror can not be considered as a perturbation of the uncorrugated waveguide and, even if the Bragg mirror supports a genuine nonleaky Bloch mode, outof-plane scattering losses are inevitable. These additional losses are due to a mode mismatch between the fundamental mode of the unperturbed waveguide and some quantity directly related to the fundamental Bloch mode of the mirror. The losses, that vary as the square of the integral overlap between the two electromagnetic modes, ${ }^{7}$ are detrimental to the performance of short cavities with a high $Q$ factor and high transmission peaks. Thus, strong corrugations required for short interaction lengths and small radiation losses required for high performance seems to be two conflicting objectives. As shown by numerical computation in Ref. 7, tapered mirrors that incorporate a series of etches whose feature dimensions vary progressively can provide short in-

${ }^{a)}$ Electronic mail: philippe.lalanne@ iota.u-psud.fr teraction lengths and low radiation losses over a broad spectral interval. In this work, we provide experimental evidence of the beneficial effect of such an approach.

Silicon-on-insulator wafers with a $0.34 \mu \mathrm{m}$ core thickness and a $1 \mu \mathrm{m}$ thick silicon dioxyde cladding are used in this work. The mirror patterning is done by electron-beam lithography with a JEOL JBX5D2U vector scan generator on a single layer poly(methylmethacrylate) (PMMA) resist spin coated on top of the sample. The electron-beam energy is 50 $\mathrm{keV}$ resulting in a $30 \mathrm{~nm}$ diameter probe beam. A modulated electron-beam dose is used to reduce proximity effects. After developing the PMMA resist, an intermediate nickel layer was electron-beam evaporated onto the surface and lifted off by dissolving the PMMA. The lift-off technique improves the selectivity and the fidelity of the pattern transfer during the reactive ion etching process. RIE of the silicon layer was then performed in a Nextral NE110 system with a $\mathrm{SF}_{6}(1 / 3)$ and $\mathrm{CHF}_{3}(2 / 3)$ gas mixture, at a pressure of $10 \mathrm{mT}(1.33$ $\mathrm{Pa})$. The etching parameters have been optimized to obtain vertical sidewalls of silicon with an etching rate of $50 \mathrm{~nm} /$ min. The silicon dioxyde cladding is used as an etch stop. After etching, the nickel mask is removed by wet chemical etching with diluted $\mathrm{HNO}_{3}$ acid. Finally, the wafer is thinned down to less than $100 \mu \mathrm{m}$ and cleaved to obtain optical facets.

Both tapered and nontapered mirrors are manufactured on the same substrate. For the sake of testing, these mirrors are incorporated into cavities. Figure 1(a) shows the cavity formed with two conventional first-order Bragg short mirrors etched into a $5 \mu \mathrm{m}$ wide rib waveguide. The mirror period is $290 \mathrm{~nm}$ and the slit width is $90 \mathrm{~nm}$. All the slits are fully etched down to the oxide layer. Figure 1(b) corresponds to the tapered cavity. As shown by the enlarged view of Fig. 1(c), the tapered mirrors are formed of one slit with a $90 \mathrm{~nm}$ width surrounded each by two tapers consisting in a series of eight slits with linearly varying widths from 40 to $80 \mathrm{~nm}$. The progressive variation of the slit width implements an 


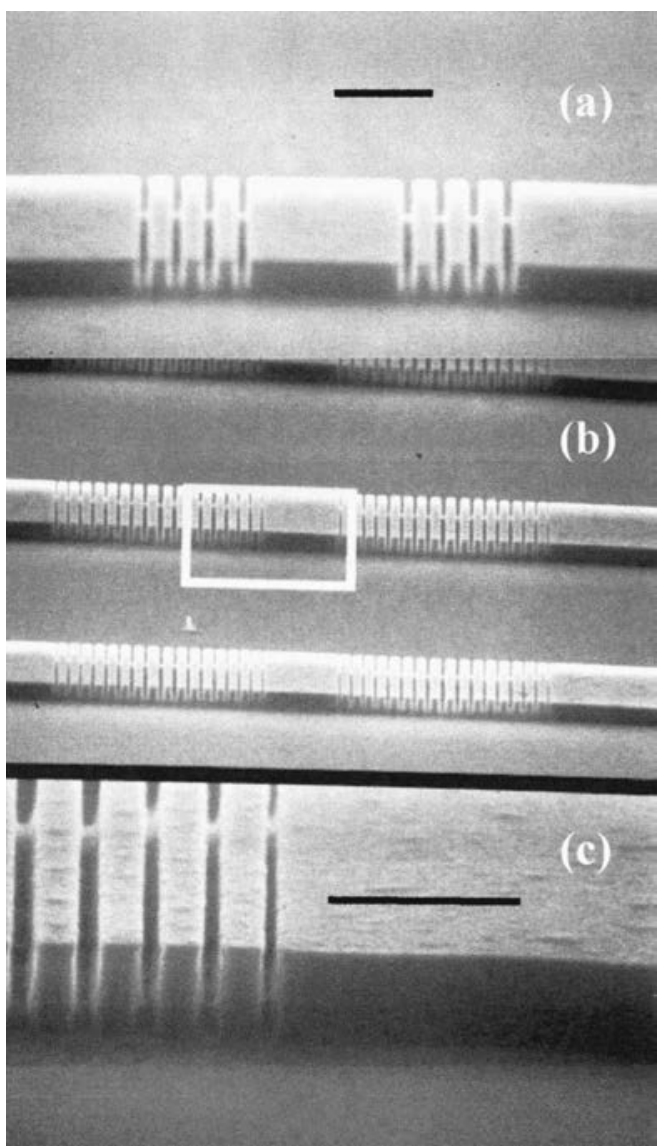

FIG. 1. Scanning electron micrographs of the microcavities. (a) cavity with nontapered mirrors. (b) cavity with tapered mirrors. (c) enlarged view of image (b) showing the taper made of a series of slits whose feature dimensions vary progressively. The horizontal black lines represent $1 \mu \mathrm{m}$.

artificial material with a graded effective index and provides an adiabatic modal transformation which lowers the mode mismatch problem. ${ }^{7}$

Transmission measurements are performed with a fiberto-fiber setup under transverse electric polarization. Constant optical power is launched from a tunable external source $(1500-1590 \mathrm{~nm})$ by a microlensed fiber directly into the rib waveguides. The transmitted power is collected by a second identical fiber and sent to a Hewlett-Packard powermeter. We checked that the tunable source was spectrally pure and mode hop free over the whole spectral range. For the sake of normalization, the transmitted power of an unpatterned $5 \mu \mathrm{m}$ large rib waveguide fabricated on the same substrate is also measured. For reproducible coupling in and out of the rib waveguides, the input and output microlensed fibers are iteratively micropositioned in order to achieve maximum transmittance.

The measured normalized transmission resonances of the cavities are shown in Fig. 2(a); the corresponding theoretical transmissions are shown in Fig. 2(b). At resonance, a sharp peak in transmission is seen. The measured values are in good agreement with theory. The transmission spectrum is calculated from dimensions measured on the actual devices by neglecting the finite lateral width of the rib waveguide. For the computation, we use a Fourier modal method. ${ }^{8}$ This method is a generalization of the rigorous-coupled-wave analysis ${ }^{9}$ to waveguide diffraction problems. It incorporates perfectly matched boundary layers in the vertical direction to
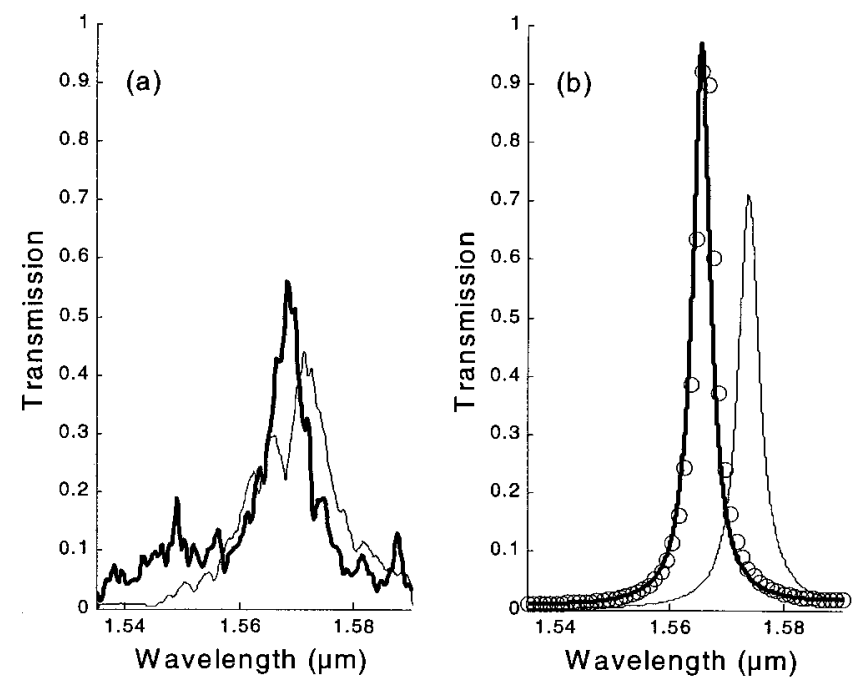

FIG. 2. Comparison of measured transmission (a) to calculated transmission (b) for the tapered cavity (bold curves) and for the nontapered cavity (thin curves). The solid curves in (b) are obtained with the one-mode model for mirror parameters $R, T$, and $\varphi$ computed with exact electromagnetic theory. Circles represent the transmission of the tapered cavity computed with exact electromagnetic theory.

accurately modelize the radiation losses. The $\approx 1 \%$ shift between calculated and measured resonance wavelengths is well within the accuracy of the scanning electron microscopy technique used to determine the device dimensions. The measured $Q$ of the tapered cavity is 200, compared with the calculated $Q$ of 410 . For the cavity with classical mirrors, the measured and calculated $Q$ 's are 110 and 370, respectively. Additionally, in Fig. 3, we show measured resonances for untapered and tapered cavities and for different cavity lengths $L$ with a $30 \mathrm{~nm}$ spacing $\delta L$. In the following, these results are used to compare the radiation losses of the tapered and nontapered mirrors.

To interpret the experimental transmission spectra, we develop an approximate model. A cavity defined by two identical mirrors is analogous to a symmetrical Fabry-Perot
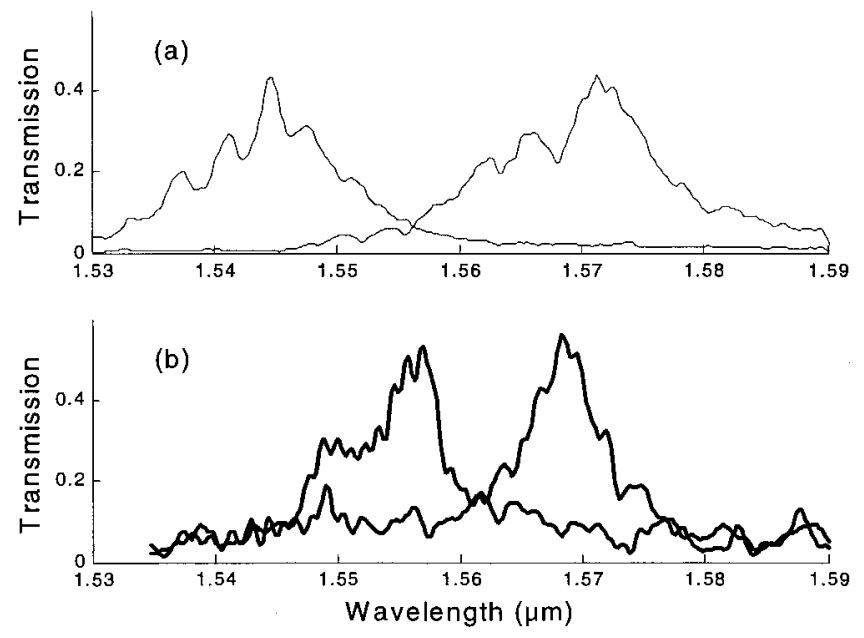

FIG. 3. Transmission of the classical cavity (a) and the tapered cavity (b) for various defect lengths $(\delta L=30 \mathrm{~nm})$. The resonance peak shifts to longer wavelengths with increasing defect length. For the same defect length, the resonance wavelength shift $\delta \lambda$ of tapered cavities is smaller than that of classical cavities because tapered cavities have effective lengths (including the penetration length into the mirror) longer than those of the nontapered ones. 
interferometer. The cavity transmission $T_{\text {cav }}$ is given by the Airy formula

$$
T_{\text {cav }}=\frac{T^{2}}{(1-R)^{2}+4 R \sin ^{2} \Phi},
$$

where $T$ and $R$ are the mirror modal transmission and reflection, and $\Phi$ is the total phase shift given by $\Phi=2 \pi / \lambda n L$ $+\varphi, \varphi$ being the mirror reflection phase and $n$ the effective index of the fundamental unpatterned waveguide. Under the assumption that $T$ and $R$ weakly depend on the wavelength, and retaining only the zeroth- and first-order terms in the Taylor series of $n(\lambda)$ and $\varphi(\lambda)$, it is easily found that the $Q$ factor of the cavity is given by

$$
Q=\frac{\sqrt{R}}{1-R}\left[\frac{2 \pi}{\lambda_{0}} n L-\lambda_{0}\left(\frac{\partial \varphi}{\partial \lambda}\right)_{\lambda_{0}}-2 \pi L\left(\frac{\partial n}{\partial \lambda}\right)_{\lambda_{0}}\right] .
$$

The first classical term corresponds to the defect phase delay. The second term is complex; it includes the additional phase delay due to the penetration depth in the mirrors, and the chromatic dispersion of the penetration depth and of the Bloch mode. The last term is related to the modal dispersion of the waveguide. As we checked through exact numerical computations, the two last terms in Eq. (2) can not be neglected. Equation (2), which relates the $Q$ factor to the mirror reflectivity, is not directly exploitable in experiments. However, it simplifies if two cavities with different defect lengths $L$ and $L+\delta L$ are measured. Provided that $\delta L$ is small enough, these two cavities resonate for the same cavity order. It is then easily shown that $(\partial \varphi / \partial \lambda)_{\lambda_{0}}=2 \pi n L / \lambda_{0}\left[1 / \lambda_{0}\right.$ $\left.-\delta L /(L \delta \lambda)-1 / n(\partial n / \partial \lambda)_{\lambda_{0}}\right]$, where $\delta \lambda$ is the resonance wavelength shift. Incorporating this expression into Eq. (2), we get

$$
\frac{\sqrt{R}}{1-R}=\frac{Q \delta \lambda}{2 \pi n \delta L} .
$$

Equation (3) allows for the determination of the modal reflectivity. The modal transmission $T$ is then determined by the peak transmission $T_{\max }$ at resonance

$$
T=(1-R) T_{\max }^{1 / 2} \text {. }
$$

Thus, the measurements of the $Q$ factor and the peak transmissions of two cavities with slightly different defect lengths allow for the determination of the mirror parameters. Equations (3) and (4) are classical since they hold for instance for bulk cavities with metallic mirrors. However, we emphasize that they are also accurate for Bragg mirrors in waveguides even for resonance frequencies close to the band edges where the penetration length varies quickly. Through exact numerical results performed with the Fourier modal method, we validated the model and found an excellent agreement for
Eqs. (1)-(4) for the tapered and the nontapered cavities and for resonance wavelengths over the full band-gap spectral region. Applying the model to the experimental results for the resonance at $\lambda \approx 1.57 \mu \mathrm{m}$, we find $R=0.79, T=0.13$ $\left(T_{\max }=0.4\right)$ for the nontapered cavity, and $R=0.79, T$ $=0.16\left(T_{\max }=0.5\right)$ for the tapered cavity. Thus, while the tapered and classical mirrors have identical modal reflection, the radiation loss $L=1-R-T$ of the tapered mirror is 0.05 , to be compared with that of the classical mirror 0.08 . This result evidences the beneficial impact of the taper, although the experimental reduction by almost a factor of 2 is smaller than that predicted by numerical computation. We believe that, because the tapered mirrors incorporate much more etches than the classical mirrors, they are relatively more sensitive to scattering due to the surface roughness of the vertical walls of the slits.

In conclusion, a silicon-on-insulator waveguide-based microcavity with first-order tapered Bragg mirrors has been realized and tested. The cavity, which incorporates four identical tapers at the four reflector extremities (overall length of $14 \mu \mathrm{m})$, has been implemented in a high-index contrast waveguide. The tapers are formed by a series of etches whose feature dimensions vary progressively and provide low insertion losses over a broad spectral interval. Because of the subwavelength scale, the segments of the taper support genuine nonleaky Bloch modes that offer nearly lossless tapering. The principle of operation is similar to that of gradient-index films used for antireflection coating. As an extension to these results for applications at a single frequency, numerical results evidencing that very short tapers optimized for operation with one or two slits appear more suitable. In this case, the tapers act as quarter-wave antireflection coatings offering the perspective of high peak transmission with an even smaller modal cavity volume.

${ }^{1}$ L. A. Coldren and S. W. Corzine, Diode Lasers and Photonic Integrated Circuits, 1st ed. (Wiley, New York, 1995).

${ }^{2}$ J. P. Zhang, D. Y. Chu, S. L. Wu, W. G. Bi, R. C. Tiberio, R. M. Joseph, A. Taflove, C. W. Tu, and S. T. Ho, IEEE Photonics Technol. Lett. 8, 491 (1996).

${ }^{3}$ J. S. Foresi, P. R. Villeneuve, J. Ferrera, E. R. Thoen, G. Steinmeyer, S. Fan, J. D. Joannopoulos, L. C. Kimerling, H. I. Smith, and E. P. Ippen, Nature (London) 390, 143 (1997).

${ }^{4}$ T. F. Krauss and R. M. De La Rue, Appl. Phys. Lett. 68, 1613 (1996).

${ }^{5}$ T. Baba, M. Hamasaki, N. Watanabe, P. Kaewplung, A. Matsutani, T. Mukaihara, F. Koyama, and K. Iga, Jpn. J. Appl. Phys., Part 1 35, 1390 (1996).

${ }^{6}$ T. F. Krauss, O. Painter, A. Scherer, J. S. Roberts, and R. M. De La Rue, Opt. Eng. (Bellingham) 37, 1143 (1998).

${ }^{7}$ M. Palamaru and P. Lalanne, Appl. Phys. Lett. 78, 1466 (2001).

${ }^{8}$ E. Silberstein, P. Lalanne, J. P. Hugonin, and Q. Cao, J. Opt. Soc. Am. A 18, 2865 (2001).

${ }^{9}$ M. G. Moharam, E. B. Grann, D. A. Pommet, and T. K. Gaylord, J. Opt. Soc. Am. A 12, 1068 (1995). 